\title{
Serious Leisure and Leisure Motivations Among Self-identified Cyclists
}

\author{
Zachary Thomas Herman \\ Appalachian State University, North Carolina, USA
}

\begin{abstract}
This study examines the leisure motivations among people who participate in cycling as a form of serious leisure. It examines what motivates cyclists to build a life around cycling. This study is a replicative study to Robert G. LaChausse's study on leisure motivations among cyclists. This study used the Serious Leisure Inventory and Measure (SLIM) created by James Gould. Participants were contacted through a variety of local and national list serves. Potential applications of this study include education, coaching, training, planning, and the creation of programs to encourage long-term cycling among bike users. The primary purpose of this study was to examine the motivations of mountain bikers, commuter cyclists, and other cyclists using the SLIM created by James Gould and the Leisure Motivation Scale.
\end{abstract}

Keywords: serious leisure, leisure motivations, cycling, cycling motivations

\section{Introduction}

Motivation behind participation in recreation activities is a growing area of study, and the implications of the results of this research have far-reaching consequences in a variety of fields, both academic and non-academic. Understanding the underlying motivations behind recreation can inform not only recreation studies, but also educational programs, city planning, design, fitness programs, tourism, exercise science, psychology, and childhood development (Shen \& Yarnal, 2010).

However, not all forms of leisure and recreation are approached in the same manner. For some people, what may start out as a simple form of recreation can slowly become an integral part of their lifestyle, self-image, and sense of self. For example, the motivations behind a day of snowboarding may be different for a person on a weekend family vacation and a person sponsored by Red Bull. The person sponsored by Red Bull to snowboard participates in what is now known as serious leisure. The recreation activity of snowboarding has shifted from a weekend diversion to a lifestyle in which massive amounts of time, money, mental energy, and physical stress have been committed. According to Gould, Moore, Karlin, Gaede, Walker, and Dotterweich (2011), serious leisure is defined as "the systematic pursuit of an amateur, hobbyist, or volunteer activity sufficiently substantial and interesting for the participant to find a career there in the acquisition and expression of a combination of its special skills, knowledge, and experience" (p. 2). Serious leisure is an activity that morphs into something that is not just a fun or satisfying way to spend time that is not dedicated to work or self-care, but a mixture of work, leisure, and self-care. Casual leisure, on the other hand, is short-lived, immediately pleasurable, and requires little skill (Gould, Moore, McGuire, \& Stebbins, 2008).

Zachary Thomas Herman, undergraduate student, Recreation Management, Appalachian State University. Email: zacharyherman6@gmail.com. 
Serious leisure is an activity that becomes a major part of a person's self-identification and lifestyle choices (Scott, 2012). Serious leisure participants are people who, within a given activity, persevere and overcome difficulties, have careers involving the activity, evince effort and utilize specialized knowledge and skills, pursue their avocations within leisure social worlds, and experience durable benefits (Scott, 2012). Serious leisure participants do not need to make money in their activity but they do need to have a similar level of commitment to the activity. What defines a serious leisure participant is a high level of physical, emotional, and financial investment.

Cycling as a form of leisure and its attached motivations has seen very little scholarship. Studies performed by LaChausse (2006) and Brown, O’Connor, and Barkatsas (2009) are the only two notable studies dealing directly with the leisure motivations of cyclists. Brown et al.'s (2009) study, Instrumentation and Motivations for Organized Cycling: The Development of the Cyclist Motivation Instrument (CMI), looked to create a tool for measuring motivations among all cyclists and was designed specifically with serious leisure conceptual understandings in mind. Brown et al.’s (2009) study looked at not only individual internal factors for motivation, but also cultural and ecological influences. Brown et al. (2009) examined five main motivation subsets: social, embodiment, physical health outcomes, self-presentation, and environmental exploration. Brown et al.'s (2009) study focused mainly on competitive cyclists. Though their research is valuable, it also overlooked non-competitive cyclists, a large part of the cycling population.

LaChausse's (2006) study was massive in both scope and scale. LaChausse's (2006) study, Motives of Competitive and Non-competitive Cyclists, examined the motivations of the cycling population looking at a more representative sample. Competitive cyclists easily fit into the mold of serious leisure participants, but non-competitive cyclists like commuters, messengers, long distance cyclers, bicycle motocross, and mountain bikers all fit the category of serious leisure without requiring that they be competitive.

LaChausse's model was based on the Motivations of Marathoners Scale (MOMS) model which was used to measure motivations of marathon runners. MOMS examined even more aspects of motivations than Brown et al.. LaChausse's survey examined health orientation, weight concern, goal achievement, competition, recognition, affiliation, coping, life meaning, and self-esteem.

The intent of this research is to replicate LaChausse's study using a different model. LaChausse's model was built on scale meant for marathoners; though the subcultures of marathoners and cyclists share similarities, they have enough differences that a model designed specifically for cyclists will yield more accurate results. This research will use the Serious Leisure Inventory and Measure (SLIM) created by James Gould. SLIM measures 12 different aspects of serious leisure and was created to be adaptable to any leisure subculture or discipline. SLIM's 12 aspects include perseverance, leisure career, significant effort, durable outcomes, individual outcomes, self-image, self-gratification, image re-creation, financial return, group outcomes, unique ethos, and identification with pursuit. The SLIM model is a dramatically more complex examination of serious leisure motivations, ideally improving the results interpreted by LaChausse.

Brown et al. failed to look at a complete population by excluding competitive cyclists. LaChausse failed to look at cycling from a serious leisure metric, ignoring the nature of cycling and long-term cyclists. This study will attempt to fill in the holes and gaps in both Brown et al.'s and LaChausse's studies. 


\section{Methods}

This research project used an online survey. The survey consisted of three parts. The first part used a simplified version of the SLIM created by Gould et al. (2008). The second part used the Leisure Motivation Scale, and the third part collected researcher-targeted demographics. The survey consisted of 63 questions: 18 Likert-type scale questions using the SLIM model which employs a 7-point scale, 40 questions from the Leisure Motivation Scale that makes use of a 5-point scale, and five demographic questions.

The survey was created on and hosted through SurveyMonkey. Participants in the study were found through various cycling organizations including: Appalachian State Cycle team, Lees-McRae Cycle team, Boone Area Cyclists, AORE List Serve, a competitive women's cyclist list serve used by the Appalachian State Exercise Science Department, and multiple personal contacts. All participants were self-identified cyclists. The survey was sent out via email to the collected participants, from February 1 to March 16. The sample size was 93 participants out of 400 contacted resulting in a $23 \%$ response rate. The survey was anonymous and totally voluntary. Data collected through the survey were analyzed using Excel and Statistical Package for Social Sciences (SPSS). This project was approved by Institutional Review Board (IRB) of Appalachian State on January 28, 2014.

\section{Results}

The data consisted of 92 respondents. Of the respondents, 24 were female, 54 were male, and 14 chose not to specify gender. Individual cycle sports were sorted, according to those who practiced them. Participants, who participated in a given sport over 10 times a month, were identified as a practitioner. All sports that had a size of less than five were removed from the data pool. The only sports with a size greater than five were commuters with $n=26$ and mountain bikers with $n=12$. Within age groups, 20-29 had an $n$ of 25, 30-39 had an $n$ of 15, 40-49 had an $n$ of 13, 50-59 had an $n$ of 13, and 60-69 had an $n$ of 8. The mean age was 37, with a median of 38, mode of 20, and a range of 53 for the ages of all participants. For income, the mean income was 52,173.00, the median was $44,000.00$, the mode was $100,000.00$, the minimum was 0.00 , and the maximum was $100,000.00$, with 67 respondents reporting income. The participants were also sorted according to their local geography, whether it was flat, mountains, or hills. Within the three classifications, flat had an $n$ of 6 , hills had an $n$ of 13 , and mountains had an $n$ of 59 .

The motivations across gender, seen in Figure 1, were all within a tenth of a point from each other. The strongest motivator among the Leisure Motivation Scale was competency with a score of 4.1 out of 5 which was more than two standard deviations above the average motivation score of 3.5.

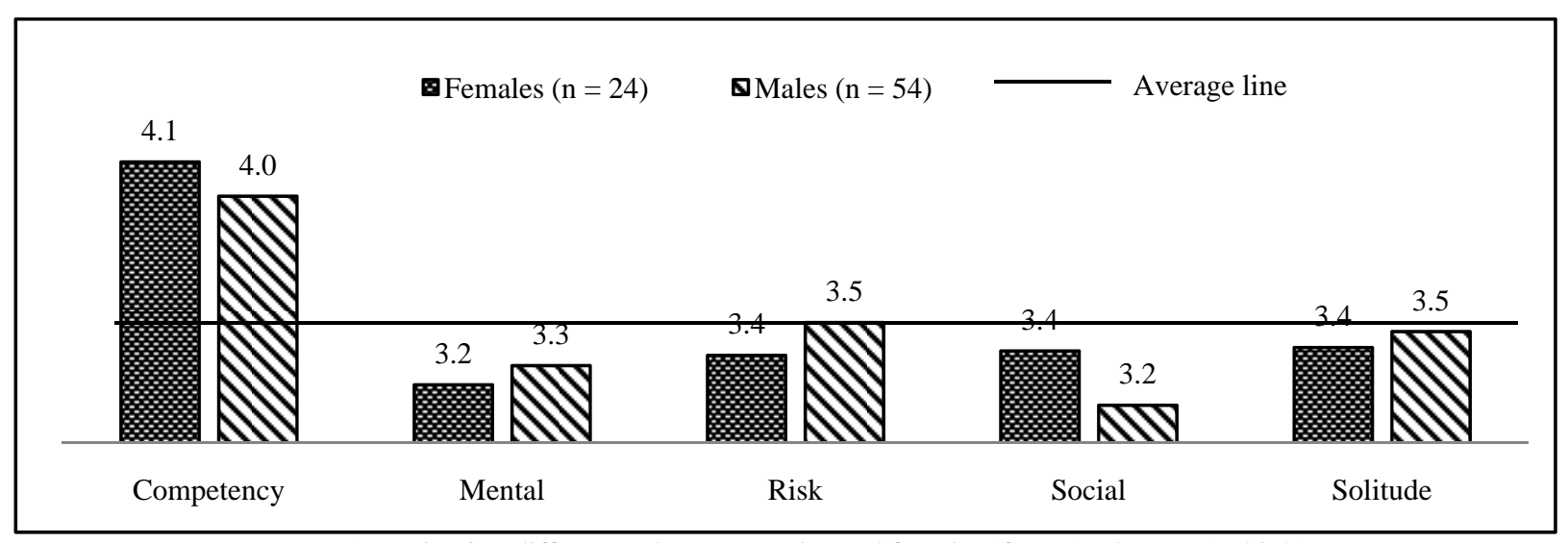

Figure 1. Motivation differences between males and females (from $1=$ low to 5 = high). 
Figure 2 shows differences in serious leisure motivation by gender. The strongest motivator by gender for serious leisure, seen in Figure 2, was "pleasure" with a score of 6.4 out of 7 and then "effort" with a score of 6.2 out of 7. Both scores in effort and pleasure were two standard deviations above the mean which was 5.5. The differences in serious leisure motivation scores by gender were also within a tenth of a point of each other.

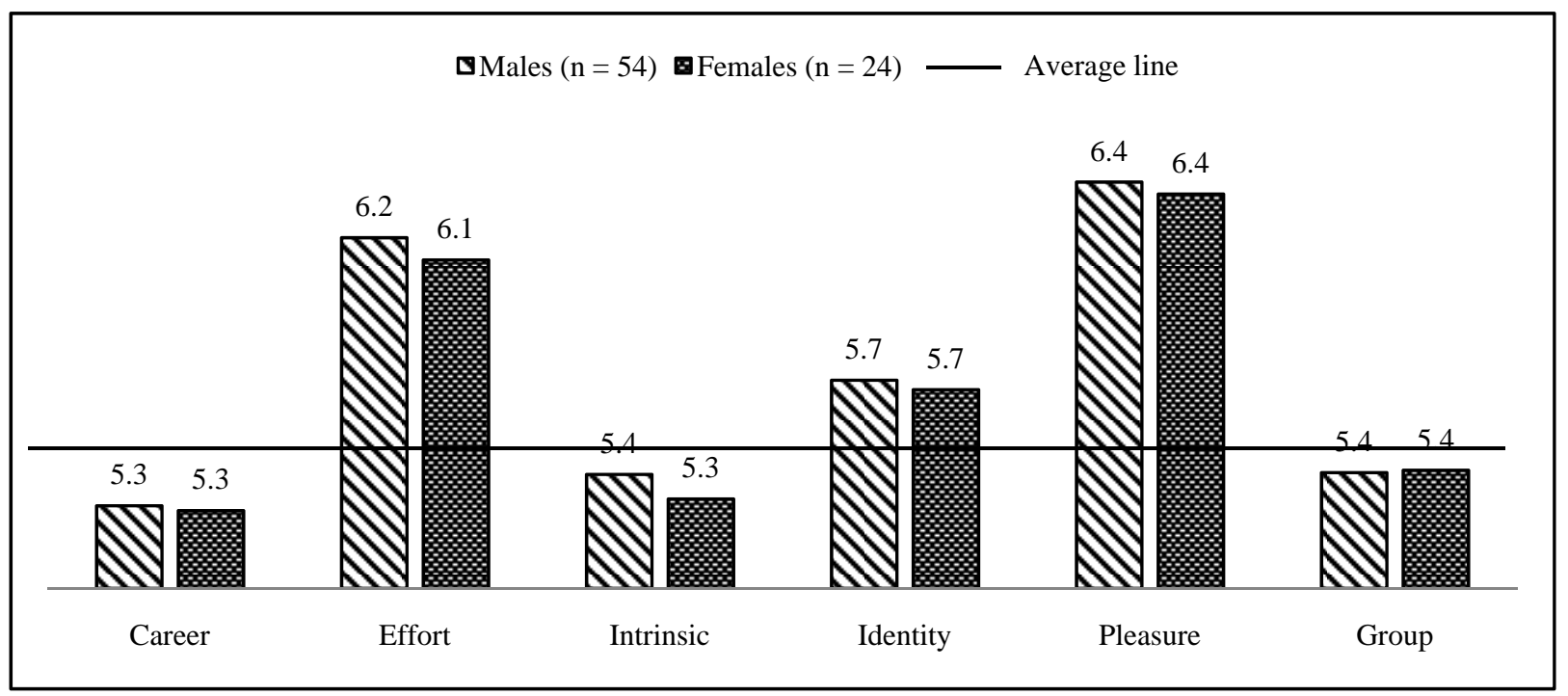

Figure 2. Serious leisure differences by gender (from 1 = low to 7 = high).

Figure 3 shows differences in leisure motivations between commuters and mountain bikers. In Figure 3, measuring differences in leisure motivations, between mountain bikers and commuters, the strongest leisure motivator, with an average score of 4.1 was competency. This is true across for all bikers, participating in the survey. Competency was also two standard deviations above the mean of 3.7. Mountain bikers had the strongest leisure motivation score of all the participants. Mountain bikers also showed strong motivations in risk seeking and social. The mountain biker motivation scores in social and risk were well above the scores for commuter and all bikers in those same categories. For both commuters and all cyclists, competency was the only leisure motivator that was high enough to be significant.

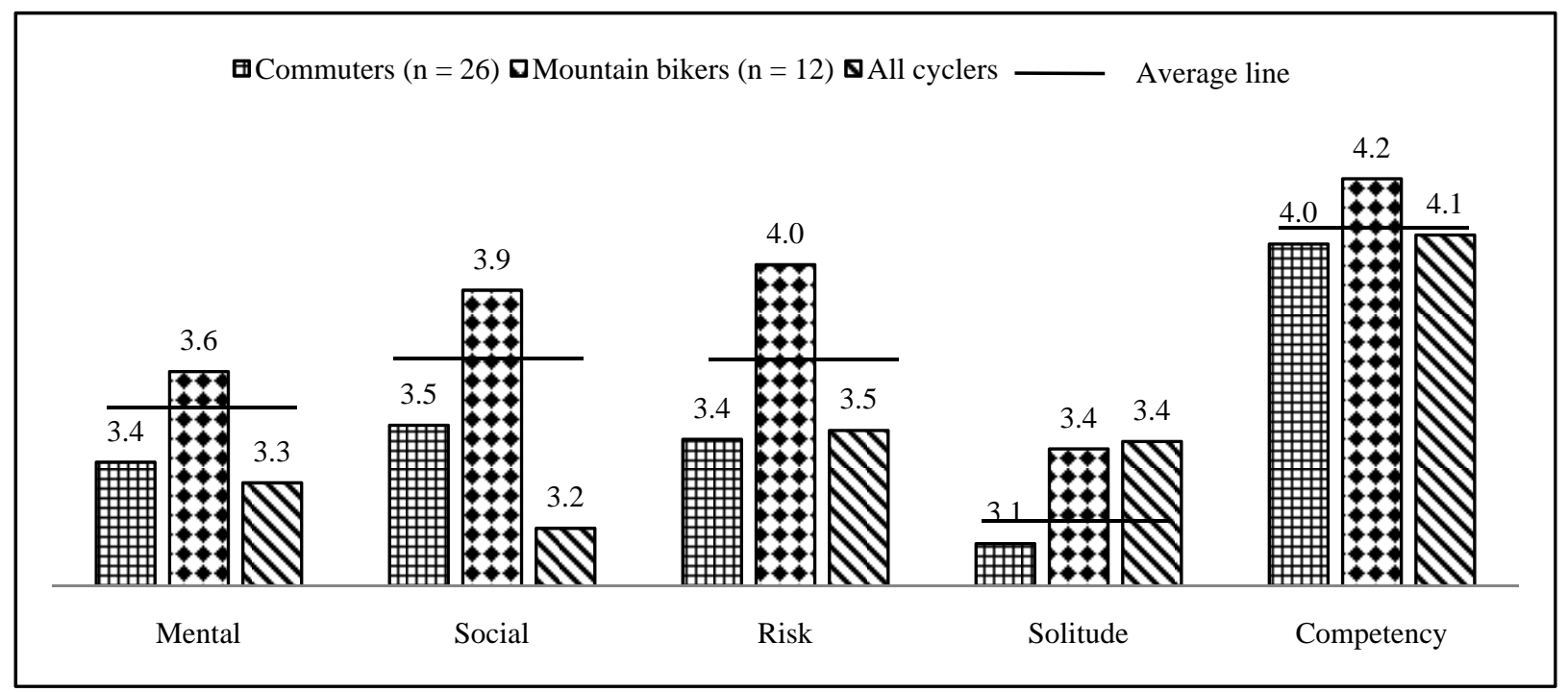

Figure 3. Motivation differences between mountain bikers and commuters (from 1 = low to 5 = high). 
Figure 4 shows the differences in serious leisure motivations between commuters and mountain bikers. The strongest serious leisure motivator between commuters and mountain bikers, as seen in Figure 4, was pleasure with a score of 6.6, followed by effort with a score of 6.4. In serious leisure motivators, mountain bikers showed a strong motivation towards group connection with a score of 6.0 out of 7 , a solid 0.6 points above all cyclists and 0.9 points above commuters.

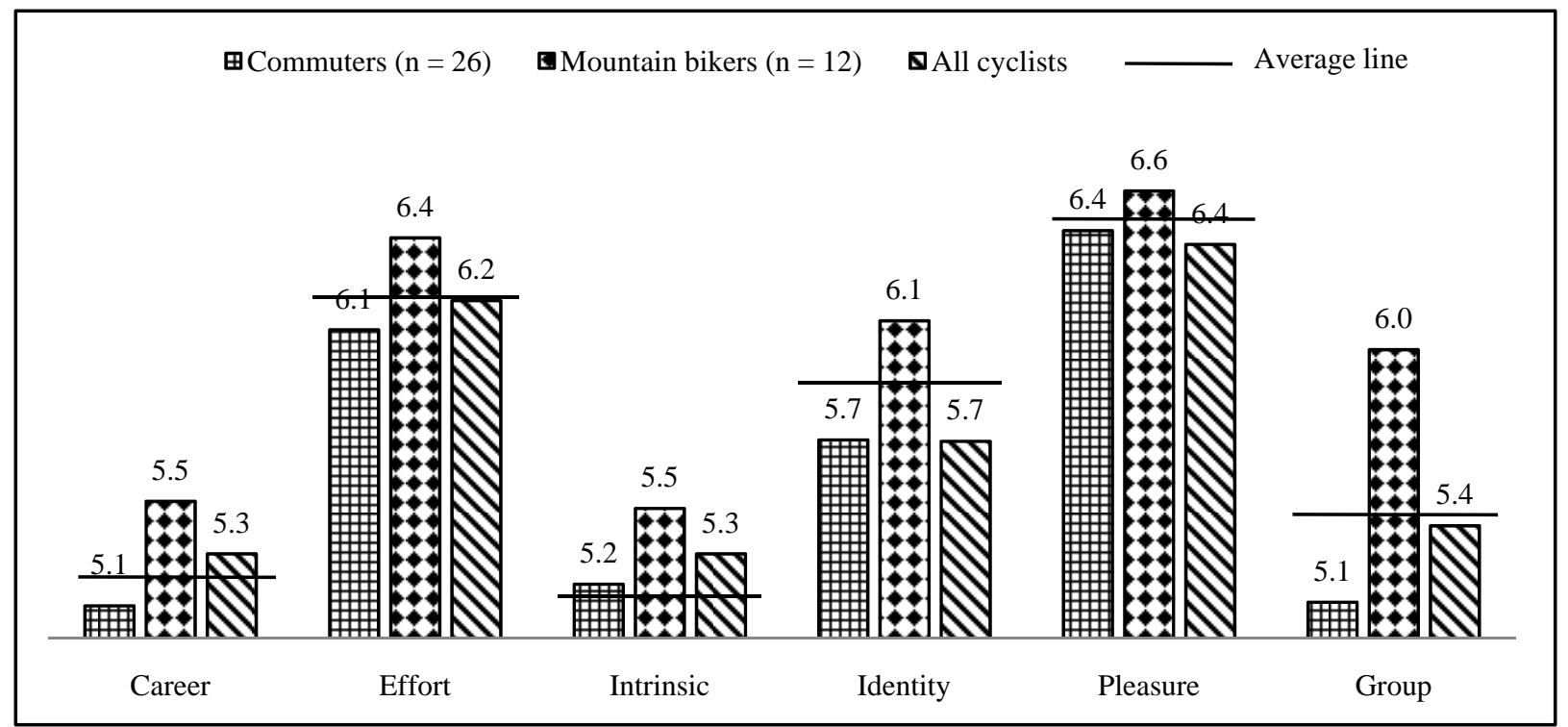

Figure 4. Serious leisure differences between commuters and mountain bikers (from $1=$ low to 7 = high).

Figure 5 shows differences in leisure motivations by income. Measuring by income, as seen in Figure 5, the strongest leisure motivator was competency with a score of 4.3, while the people in the lowest income group making between 0 and 30,000 a year had the strongest motivations across groups scoring well above the mean in all categories. People in the lower SES group, making between 0 and 30,000 a year, were consistently more motivated in all categories of leisure motivation.

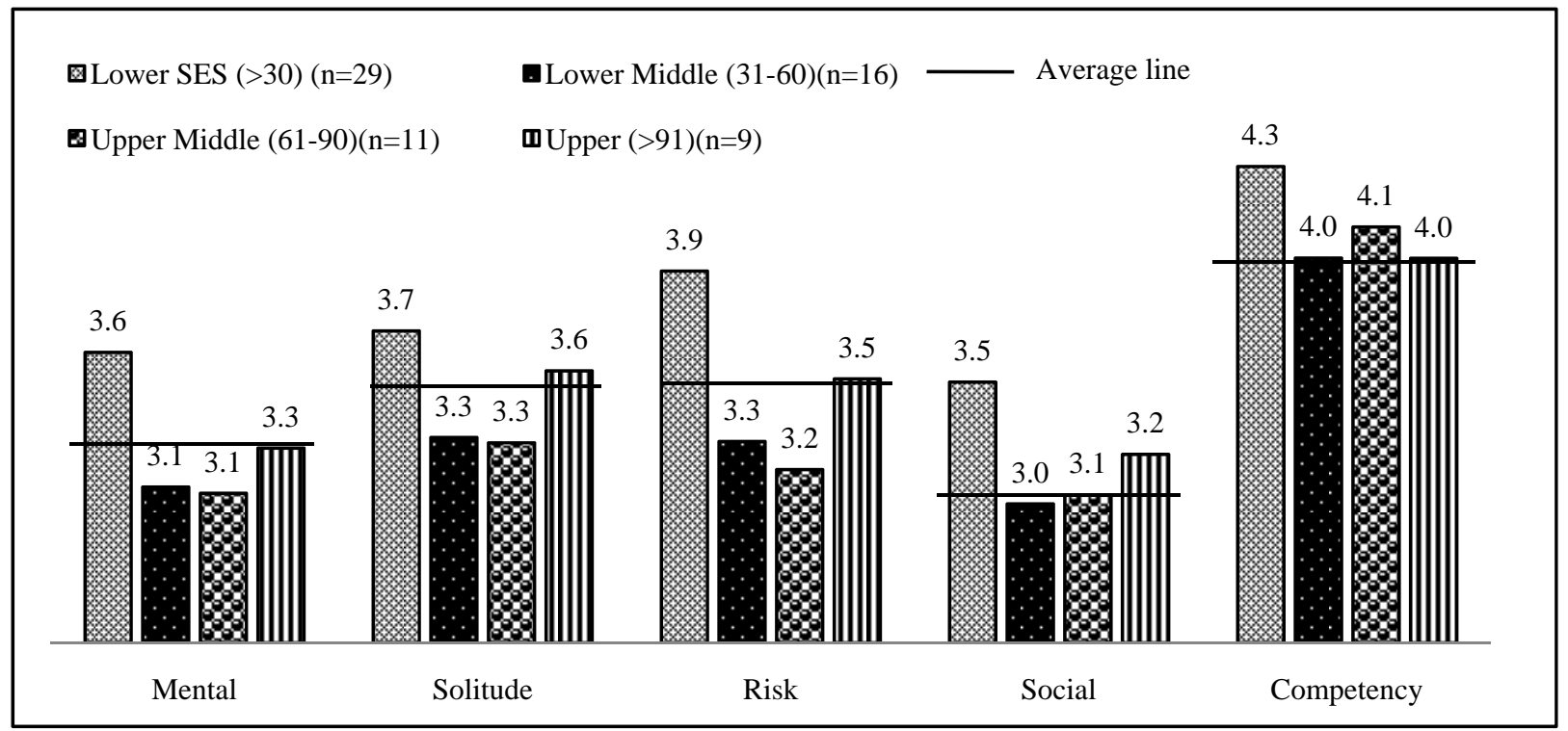

Figure 5. Motivation differences by income (from 1 = low to 5 = high). 
Figure 6 shows the serious leisure motivations among different income groups. The strongest serious leisure motivator by income, seen in Figure 6, was pleasure with a score of 6.5, followed by effort with a score of 6.3. Figure 6 shows that pleasure and effort are the two strongest motivators for cyclists, when looking at the differences among different income groups.

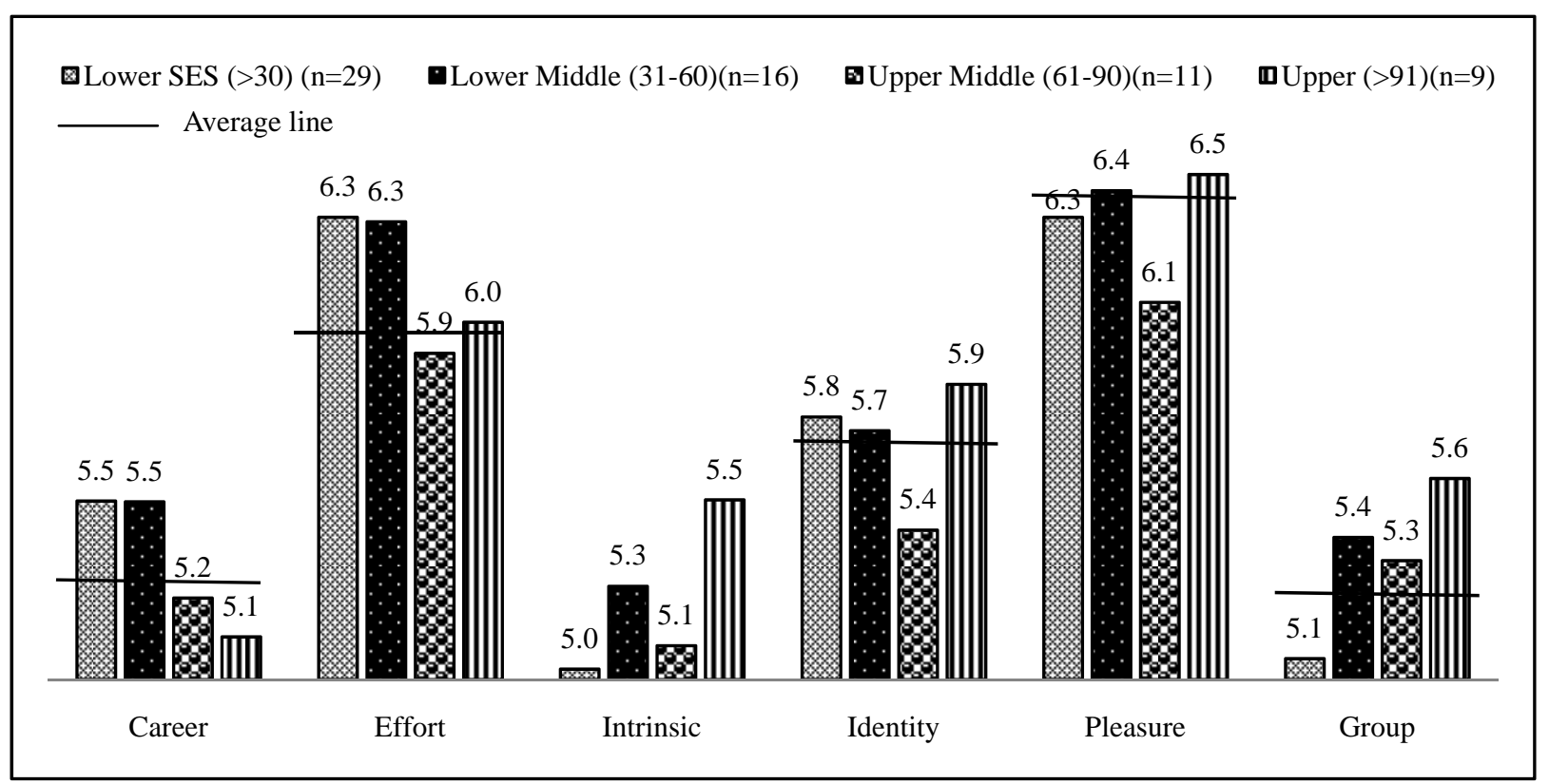

Figure 6. Serious leisure differences by income (from 1 = low to 7 = high).

Figure 7 shows the serious leisure motivations across age groups. The serious leisure motivations across age groups, seen in Figure 7, were typical with pleasure and effort being the strongest motivators scoring 6.4 and 6.1 respectively. Figure 7 also shows a steady increase in serious leisure motivation as age increases. Participants aging between 60 and 69 were equal to or more motivated than participants aging between 20 and 29.

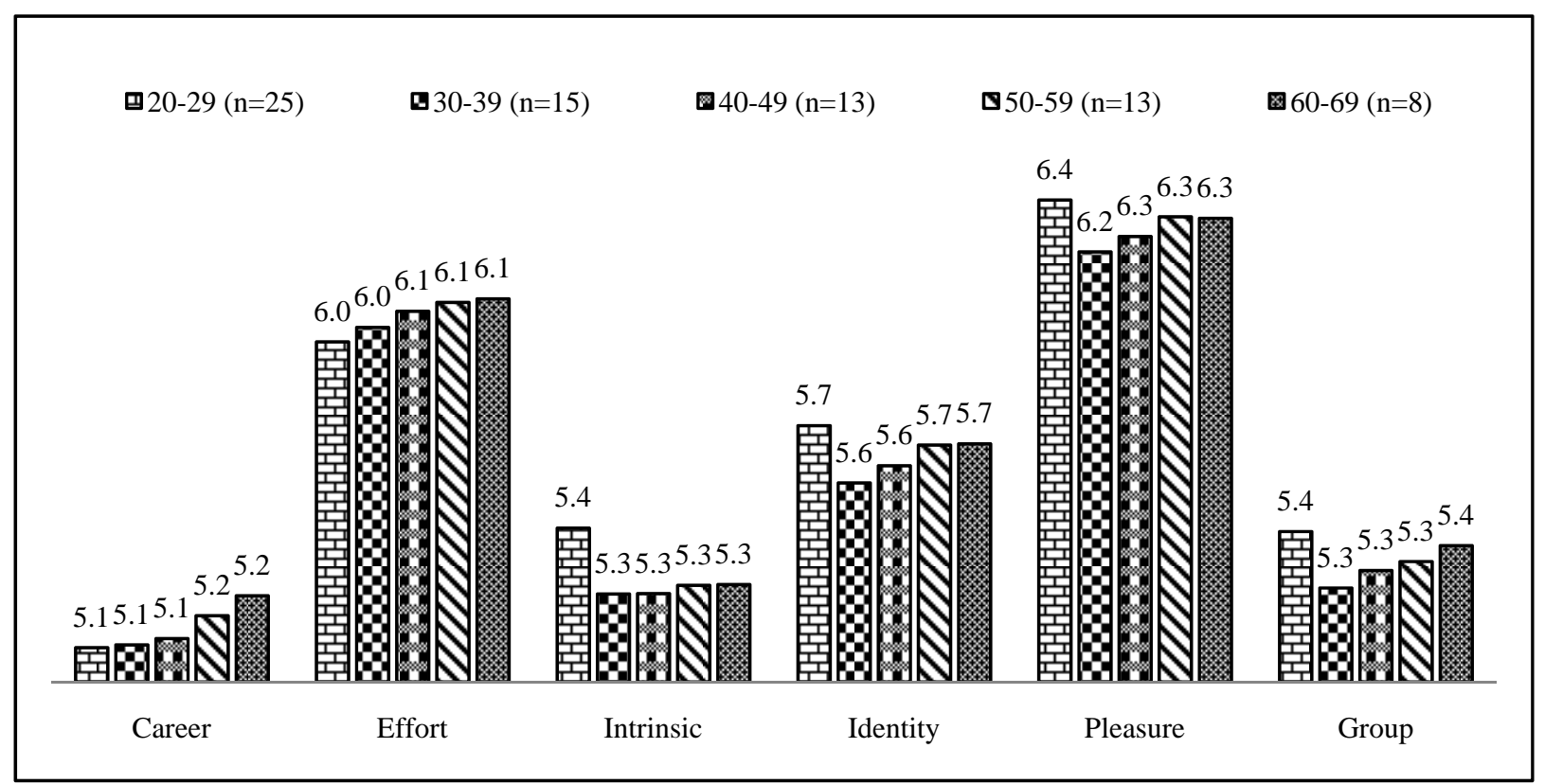

Figure 7 . Serious leisure differences by age (from 1 = low to 7 = high). 


\section{Conclusion}

Cycling should be studied as a recreational activity with very strong serious leisure potential. When looking at serious leisure motivations by age, there was a steady increase in motivation as age increased. As cyclists get older, their motivation to continue cycling will increase. Serious leisure activities are activities whose motivation increases in intensity with time. Across all age groups, the strongest serious leisure motivator was pleasure followed by a sense of effort.

For all cyclists, a sense of skill development is the strongest motivator; this is demonstrated by the fact that for all cyclists, the strongest leisure motivator was a sense of competency. The strongest serious leisure motivators for all cyclists were a sense of pleasure followed closely by effort. Cyclists want to feel competent when they ride their bikes, feel effort expended when they ride and want to have a sense of pleasure.

Compared with mountain bikers and the population as a whole, commuters have lower scores for both serious leisure and leisure motivation. This is most likely due to the fact that cycling for commuters is a mix of leisure and also can serve as a work activity. Commuting for a cycler is not pure recreation, so the motivations for doing it are not totally leisure motivated, or leisure related. Despite their overall lower motivation, a sense of competency, effort, and pleasure were still the strongest motivators for commuters. Commuters showed almost no motivation in terms of solitude or group connection. Commuters do not care if they are riding alone, in a group, or identified as a commuter or commuter community.

Mountain bikers, compared with the total population and commuters, had the highest motivation score across the board. Mountain bikers were highly motivated by a sense of risk and social connection. This was not really surprising, as mountain biking is a high sensation-seeking activity, and social connection is very important for mountain biking safety. The highest motivation scores for mountain bikers were the same as commuters and the general population. Mountain bikers want a sense of skill development; they want lots of effort, pleasure, and a sense of being a competent mountain biker.

Almost totally across all demographics, a sense of competency was the highest leisure motivator. Cyclists want a sense of skill development and a sense of competency. This sense of competency transfers over to the serious leisure motivators as well. Pleasure and effort were the highest serious leisure motivators across income, age, gender, and discipline. Cycling provides a mix of pleasure and effort, which provides a sense of flow and skill development.

The differences in motivations for men and women to participate in cycling were not very different. The scores for both leisure motivation and serious leisure were never more than a tenth of a point in difference. The major motivator for both men and women was a sense of competency, or skill development. The motivators that help turn cycling into serious leisure across both genders and sports were a sense of pleasure and then accomplishment through effort expended, or a sense of competency and flow.

\section{Program Recommendations}

The research suggests that the strongest way to motivate people to participate in cycling is to give them a sense of skill development and encourage competency. Clinics on safe commuting techniques, distance riding, as well as trail riding and mountain biking are all likely ways to encourage skills. There is an assumption that cycling is as easy as riding a bike, however, this is not the case. People who ride the bikes are motivated strongest by the opportunity to become skilled riders. 
Commuting can be an intimidating undertaking especially in locations that lack proper cycle commuting infrastructure. Allowing for practice spaces for commuters, and alternate or cycle only routes through and to town, will allow for cyclers to gain skill in commuting. Mentoring programs or organized commuter group ride programs would also allow for less skilled commuters to learn from and feel safe with skilled, experienced commuters. Programs on rules of the road for cyclers and how to be an aggressive or defensive commuter will also help motivate people to turn to cycling for commuting.

Mountain bikers need a sense of group connection; this is most likely due to safety needs if an accident happens on trial. Organizing group mountain biking rides with mixed skill levels would provide both a sense of group connection and an opportunity for a sense of competency. We should mark and grade trails for mountain bikers, so they know what skill level is expected. Clinics on mountain biking techniques, tricks, and skill development will also help motivate people to participate in mountain biking.

Cycling is a task that is assumed to be simple and easy, but this does not seem to be true. For people to feel motivated to ride, they need to feel competent and be able to improve their skills. Ways to improve skills and competency are crucial to motivating people to ride their bikes.

In programs focusing on continuing competition, a sense of social competition among teammates could augment a sense of desire for self-improvement and encourage long-term commitment to the team. It is even suggested in research done by Corbett, Barwood, Ouzounoglou, Thelwell, and Dicks (2012) that a sense of team competition in training can increase time trial performance in the race more than with just self-paced time trial training.

Two studies done by Eyck, Geerlings, Karimova, Meerbeek, Wang, IJsselsteijn, De Kort, Roersma, and Westerink (2006) and IJsselsteijn, De Kort, Bonants, De Jager, and Westerink (2004) both suggest that even virtual sense of social connection mixed with a sense of self-improvement can help increase motivation. Both studies were done on the use of virtual coaches on competitive cyclist performance. Both studies showed an increase in performance post virtual training session when a virtual coach was introduced to the program.

\section{Recommendations for Future Research}

Future research will require a larger, more diverse, and more representative sample of cyclists, across region, cycling discipline, income, age, and gender. The sample from this research has a relatively small sample size compared with similar studies, which often reach between 1,200 and 1,500 participants. The respondents were also dispersed almost exclusively in the southern Appalachian mountain region, creating an unrepresentative sample. This study also has a self-selection bias, so creating a random sample of cyclists is needed.

Future research should use an expanded version of the SLIM for a more accurate view of serious leisure motivators. Future research should also use Optimal Level of Arousal scale to look at possible connections to a sense of "flow" satisfaction among cyclists, and possibly examine if serious leisure as a form of leisure is connected to personality traits. In addition, future research should look for self-identified cyclists and possible connection to personality traits, examine cycling among older populations with possible increasing physical limitations, and see if they change their leisure needs or if their motivations change.

Possible qualitative research should look for cyclists who self-identify themselves as cyclists. Qualitative research should look at stories on how cycling was introduced. A project with a qualitative look at cyclists between the ages of 60 and 100 would be looked at when they started cycling and important moments in their 
cycling carrier. A qualitative study would also include interviews with sponsored cyclists, both competitive and non-competitive, looking for connections in how they think about cycling and how they entered the sport of cycling.

\section{References}

Brown, T. D., O’Connor, J. P., \& Barkatsas, A. N. (2009). Instrumentation and motivations for organised cycling: The development of the cyclist motivation instrument (CMI). Journal of Sports Science and Medicine, 8(2), 211-218.

Corbett, J., Barwood, M. J., Ouzounoglou, A., Thelwell, R., \& Dicks, M. (2012). Influence of competition on performance and pacing during cycling exercise. Medicine and Science in Sports and Exercise, 44(3), 509-515.

Eyck, A., Geerlings, K., Karimova, D., Meerbeek, B., Wang, L., IJsselsteijn, W., de Kort, Y., Roersma, A., \& Westerink, J. (2006). Effect of a virtual coach on athletes’ motivation. In Persuasive technology (pp. 158-161). Berlin, Heidelberg: Springer.

Gould, J., Moore, D., Karlin, N. J., Gaede, D. B., Walker, J., \& Dotterweich, A. R. (2011). Measuring serious leisure in chess: Model confirmation and method bias. Leisure Sciences: An Interdisciplinary Journal, 33(4), $332-340$. doi:10.1080/01490400.2011.583165

Gould, J., Moore, D., McGuire, F., \& Stebbins, R. (2008). Development of the serious leisure inventory and measure. Journal of Leisure Research, 40(1), 47-68.

IJsselsteijn, W. A., De Kort, Y. A. W., Bonants, R., De Jager, M., \& Westerink, J. H. D. M. (2004). Virtual cycling: Effects of immersion and a virtual coach on motivation and presence in a home fitness application. Proceedings of Virtual Reality Design and Evaluation Workshop (pp. 22-23).

LaChausse, R. G. (2006). Motives of competitive and non-competitive cyclists. Journal of Sport Behavior, 29(4), 304-314.

Scott, D. (2012). Serious leisure and recreation specialization: An uneasy marriage. Leisure Sciences: An Interdisciplinary Journal, 34(4), 366-371. doi:10.1080/01490400.2012.687645

Shen, X. S., \& Yarnal, C. (2010). Blowing open the serious leisure-casual leisure dichotomy: What's in there? Leisure Sciences: An Interdisciplinary Journal, 32(2), 162-179. doi:10.1080/01490400903547179 